\title{
Combined effect of UV-radiation and triflumuron on the progeny of Alphitobius diaperinus (Panzer)(Coleoptera: Tenebrionidae) at different storage period
}

\author{
Mahmuda Begum ${ }^{1}$, Selina Parween ${ }^{2}$ \& Saiful Islam Faruki ${ }^{2}$ \\ ${ }^{1}$ Institute of Biological Sciences, Rajshahi University, Rajshahi-6205, Bangladesh \\ ${ }^{2}$ Department of Zoology, Rajshahi University, Rajshahi - 6205, Bangladesh
}

\begin{abstract}
Eggs of the lesser mealworm, Alphitobius diaperinus (Panzer) were exposed to UV-rays of 254nm wavelength for different durations viz. 1, 2, 4 and 8 minutes. After seven days of hatching, larvae of each exposure were reared separately on triflumuron $(1 \times 10-3 \mathrm{mg} / \mathrm{kg})$ treated food and observed the population of various developmental stages e.g., egg, larval, pupal and adult at storage periods of 3-, 6- and 9-months. UVrays and triflumuron treatments deleteriously reduced the populations of all the developmental stages of A. diaperinus. Egg and pupal populations of the beetle were adversely influenced by the storage periods also. Complete reduction of A. diaperinus populations was achieved from 6-9 months storage periods, when eggs were exposed to UV-rays for 8 minutes.
\end{abstract}

Key words: Mealworm, UV-rays, IGR, progeny production

\section{Introduction}

The lesser mealworm or darkling beetle, Alphitobius diaperinus (Panzer) is a cosmopolitan pest (Salin et al., 2003) of poultry (Pfeiffer \& Axtell, 1980), and a minor pest of stored-products (Hinton \& Corbet, 1975; Ichinose et al, 1980). Food-borne diseases in human being due to Salmonella infected poultry is also caused by $A$. diaperinus (McAllister et al., 1994). The beetle also causes human allergy (Schroeckenstein et al., 1990).

Management of insect pests in present time, is avoiding the use of residual chemical pesticides due to a number of problems created by them to human and environment. The use of physical and bio-control agents, and insect growth regulators (IGRs) are increasingly used for pest management in agriculture, grain and cereal stores. Both UV-ray (Calderon et al., 1985; Faruki \& Khan, 1993; Faruki, 2005; Faruki et al., 2005, 2007) and IGRs (Thomas \& BhatnagarThomas, 1968; Fox, 1990) have been reported as potential agents for controlling stored-products insects.

The present research was designed to observe the potentiality of UV-radiation in controlling the population of $A$. diaperinus when the eggs were irradiated and 7-day old larvae from irradiated eggs were reared on triflumuron (an IGR) treated larval food.

\section{Materials and Methods}

Culture of A. diaperinus: Adults of A. diaperinus were collected from grain shops. In laboratory, the beetles were kept in $500 \mathrm{ml}$ glass beaker containing the standard foodmedium (19 flour:1 Brewer's yeast) (Park \& Frank, 1948). Few slices of fresh potatoes were placed insided the beaker to keep the food moist. The potatoes were replaced with fresh slices at every 3-5 days. The food medium was changed every week to avoid conditioning. The culture was kept in an incubator at $30 \pm 0.5^{\circ} \mathrm{C}$ without controlling light and humidity.

Experimentation: Eggs were collected from subcultures of $A$. diaperinus and kept in clean glass Petridishes (90mm). Eggs of 24h old were exposed to UV-radiation from a UV-lamp (15W germicidal lamp, GE15T8)(F.G. Bode \& Co. Gmbh, Germany) with 254nm wavelength. For irradiation Petridish with eggs was placed on the surface $\left(20.5 \mathrm{~cm}^{2}\right)$ apart $12 \mathrm{~cm}$ from the UV lamp and exposed for 1, 2, 4 and 8min, and 100 eggs were used for each exposure period. The eggs were then kept separately in glass beakers containing $100 \mathrm{~g}$ of freshly prepared standard food. Top of the beakers were covered with fine cloth, tied with rubber band and kept at the culture temperature. Seven days after hatching, the old food was replaced with triflumuron ( $1 \times 10^{-3} \mathrm{mg} / \mathrm{kg}$ ) treated food. The number of eggs, larvae, pupae and adults were counted after 3 , 6 and 9 months. After each 60 days $100 \mathrm{~g}$ of fresh untreated food was added to each beaker to avoid food scarcity for overcrowding and conditioning of the food by the beetles (Mondal \& Port, 1995). Simultaneously, equal number of non-irradiated eggs were reared on untreated standard food throughout the experiment, at same temperature, as control. The experiment was replicated for three time.

The percentage reduction in population (progeny) of the treated batch compared to the Control batch was determined using the formula as suggested by Mian \& Mulla (1982). The formula is as follows:

Percentage reduction of progeny $=100(1-t / c)$, where, $\mathrm{t}=$ number of progeny in treated batch, and $\mathrm{c}=$ number of progeny in Control batch. 


\section{Results and Discussion}

Total progeny production of $A$. diaperinus was severly affected when the eggs were exposed to UV-radiation and the early larvae started to feed on triflumuron treated food for first two months (Table 1). The percentage of reduction of progeny was increased with the exposure time, and $100 \%$ reduction was observed in the population which were exposed to UV-radiation for 8 minutes. Even at 2 minutes exposure the percentage reduction from 3-9 months was calculated as 82.58 to $90.04 \%$. Reduction of population at all exposure period varied significantly $\left(\mathrm{F}_{4 / 8}=82.46, \mathrm{P}<\right.$ $0.001)$, whereas storage periods hava no effect on population $\left(\mathrm{F}_{2 / 8}=3.75, \mathrm{P}>0.05\right)($ Table 2$)$.

Egg production of the irradiated and triflumuron fed beetles was reduced significantly in all the batches receivings radiation for different period $\left(\mathrm{F}_{4 / 8}=17.48, \mathrm{P}\right.$ $<0.001$ )(Table 2). Percentage of egg production was decreased with increasing experimental period, which was also differed among each storage periods $\left(\mathrm{F}_{2 / 8}=\right.$ $7.24, \mathrm{P}<0.05)$. Complete reduction $(100 \%)$ of eggproduction was observed even after 3 months in the batch receiving radiation for 8 minutes. After 9 months $100 \%$ reduction of egg-production was observed in the batch which was irradiated for 2 minutes.

Reduction of larval population was comparatively less than reduction of eggs. At all storage periods the batch receiving 8 minutes radiation produced no larvae (Table 1). Reduction of larvae varied from $46.03 \%$ (at 1 minute exposure after 9 months) to $87.50 \%$ (at 2 minute exposure after 6 months). The larval population significantly differed between the exposure periods $\left(\mathrm{F}_{4 / 8}=18.72, \mathrm{P}<0.001\right)$.

The pupal population was severely affected by UVradiation and larval feeding on triflumuron. After 6 months no pupae were obtained at any batch of the beetles receiving radiation longer than 1 minute (Table 1). After 3 months the reduction of pupae was determined from $60.00-86.00 \%$ when exposed to UVrays for 1- 4 minutes. The effects of UV-radiation and triflumuron significantly differed among the Control and the treated batches $\left(\mathrm{F}_{4 / 8}=41.42, \mathrm{P}<0.001\right)$ and also in all storage periods $\left(\mathrm{F}_{2 / 8}=7.10, \mathrm{P}<0.05\right)$ (Table 2$)$.

However, the adult population was recovered more than the pupal population in the batches receiving 1-8 minutes UV-radiation (Table 1). Percentage reduction of adult progeny was 100 in the batch which was exposed for 4 minutes (after 9 months) and 8 minutes (after 6 months). Minimum reduction in adult population (0.97-39.78\%) was observed in the batch receiving 1 minute exposure to UV-rays for 3-9 months storage periods. Reduction of adult population was significantly differed among the irradiation period $\left(\mathrm{F}_{4 / 8}=36.42, \mathrm{P}<0.001\right)$.

Result shows that UV-exposure of 24h old eggs and subsequent feeding on triflumuron treated food effectively controlled progeny production of $A$. diaperinus in 9 months experimental period. Treatments to the initial population resulted in $100 \%$ reduction of egg production after 9 months when irradiated for 2 and 4 minutes; no eggs were found in the population exposed for 8 minute exposure after 3 months. So, exposure of 24h old eggs to UV-rays for 8 minute and subsequent larval feeding on low dose $(1 \mathrm{x}$ $10^{-3} \mathrm{mg} / \mathrm{kg}$ ) triflumuron treated food, gave total control of A. diaperinus population. Larvae of different instars were observed in treated batches even after 9 months, at 2-4 minutes irradiated batches, but pupal recovery was $100 \%$ reduced after 6 -months in these batches. A smaller percentage of larvae ultimately completed metamorphosis and adults were observed after completion of 3-9 months storage. Pupal stage of these larvae may be escaped during data collection after 3-, 6- and 9-months. However, UV-radiation to eggs and larval feeding on triflumuron have a prolonged combined action on the growth and development of $A$. diaperinus. Faruki et al. (2007) reported that egghaching and adult emergence were significantly reduced in Tribolium castaneum, T. confusum and Cadra cautella due to exposure of eggs to UV-rays.

UV-exposure of larvae (Faruki et al., 2005) and pupae of A. diaperinus (Parween et al., 2004) significantly reduced the reproductive potentiality of these beetles and fly species. Faruki (2005) noted significantly reduced adult emergence due to exposure of $T$. castaneum larvae to UV-rays. Workers like Calderon et al. (1985) and Faruki \& Khan (1993) reported that UVrays can be used to suppress the population of insects.

Moreover, triflumuron is an excellent larvicide at low doses (Mian \& Mulla, 1982; Parween, 2003); effectively suppress growth and development (Eisa et al., 1984; Begum et al., 2000; Faruki et al., 2002); and impair reproduction (Parween et al., 2001; Akhtar et al., 2003; Begum et al., 2003; Parween, 2004) in different stored products insect species.

Reseults of the present experiment revealed that UVradiation at 2-4 minutes to young eggs and subsequent larval feeding on low dose $\left(1 \times 10^{-3} \mathrm{mg} / \mathrm{kg}\right)$ of triflumuron-treated food can effectively suppress the population of $A$. diaperinus up to 9 months of storage period. Thus, it may conclude that both UV-radiation and low dose of triflumuron can be apply in the grain and cereal stores as safe and effective method to control the storage pests that might ensure better and hygienic quality of food for human consumption.

Acknowledgements: Authors remain grateful to the Institute of Biological Sciences and Ministry of Science and Technology, Government of the Peoples Republic of Bangladesh for financial support. Thanks are also due to the Department of Zoology, Rajshahi University for providing irradiation facilities. 
Table 1. Progeny reduction (\%) at various developmental stages of $A$. diaperinus at different storage period after exposure of eggs to UV-rays and triflumuron-treated larval food

\begin{tabular}{|c|c|c|c|c|}
\hline \multirow{2}{*}{$\begin{array}{c}\text { Exposure } \\
\text { periods (min) }\end{array}$} & \multirow{2}{*}{$\begin{array}{c}\text { Developmental } \\
\text { stages }\end{array}$} & \multicolumn{3}{|c|}{$\begin{array}{c}\text { Progeny reduction (\%) after storage } \\
\text { period }\end{array}$} \\
\hline & & 3 months & 6 months & 9 months \\
\hline \multirow{5}{*}{1} & Egg & 53.48 & 41.66 & 70.58 \\
\hline & Larva & 54.05 & 72.91 & 46.03 \\
\hline & Pupa & 60.00 & 87.50 & 94.44 \\
\hline & Adult & 39.78 & 16.38 & 0.97 \\
\hline & Egg to adult & 45.60 & 40.17 & 29.35 \\
\hline \multirow{5}{*}{2} & Egg & 72.09 & 80.55 & 100 \\
\hline & Larva & 72.97 & 56.25 & 73.01 \\
\hline & Pupa & 86.60 & 100 & 100 \\
\hline & Adult & 72.47 & 90.51 & 97.08 \\
\hline & Egg to adult & 86.48 & 82.58 & 90.04 \\
\hline \multirow{5}{*}{4} & Egg & 81.39 & 88.88 & 100 \\
\hline & Larva & 64.86 & 87.5 & 85.71 \\
\hline & Pupa & 86.60 & 100 & 100 \\
\hline & Adult & 95.16 & 96.55 & 100 \\
\hline & Egg to adult & 88.51 & 93.76 & 95.52 \\
\hline \multirow{5}{*}{8} & Egg & 100 & 100 & 100 \\
\hline & Larva & 100 & 100 & 100 \\
\hline & Pupa & 100 & 100 & 100 \\
\hline & Adult & 98.38 & 100 & 100 \\
\hline & Egg to adult & 98.98 & 100 & 100 \\
\hline
\end{tabular}

Table 2. Analysis of variance on progeny of different developmental stages of $A$. diaperinus obtaining from eggs exposed to UV-rays and triflumuron-treated larval food for 3-9 months storage periods

\begin{tabular}{|c|c|c|c|c|c|c|}
\hline \multirow{2}{*}{ Sources } & \multirow{2}{*}{ df } & \multicolumn{5}{|c|}{ F-values for A. diaperinus progenies } \\
\cline { 3 - 7 } & & egg & larva & pupa & adult & egg to adult \\
\hline Exposure periods & 4 & $17.48^{* *}$ & $18.72^{* *}$ & $41.42^{* *}$ & $36.42^{* *}$ & $82.46^{* *}$ \\
Storage periods & 2 & $7.24^{*}$ & $2.07^{\mathrm{NS}}$ & $7.10^{*}$ & $2.3^{\mathrm{NS}}$ & $3.75^{\mathrm{NS}}$ \\
Error & 8 & & & & & \\
\hline Total & 14 & & & & & \\
\hline
\end{tabular}

Note: $* \mathrm{P}<0.05,{ }^{* *} \mathrm{P}<0.001$, NS $=$ non-significant

\section{References}

Akhtar, F., Islam, S. \& Parween, S. 2003. Effect of an IGR, triflumuron on the heritability of reproductive attributes in the pulse beetle, Callosobruchus maculatus (Fab.)(Coleoptera: Bruchidae). Bangladesh j. zool. 31(1): 135-138.

Begum, M., Faruki, S.I, Mondal, K.A.M.S.H, Parween, S., Islam, W. \& Hussain M. 2000. Effect of egg treatment with triflumuron on the development of Tribolium castaneum (Herbst) (Coleoptera : Tenebrionidae). J. biosci. 8: 7-12.

Begum, M., Mondal, K.A.M.S.H., Parween, S., Faruki, S.I. \& Hussain, M.M. 2003. Effect of treatment of eggs with triflumuron on oviposition and fertility in Alphitobius diaperinus Panzer (Coleoptera: Tenebrionidae). Int. Pest Cont. 45 (3): 145-147.

Calderon, M., Bruce, W.A. \& Leecsh, L.G. 1985. Effect of UV radiation on eggs of Tribolium castaneum. Phytoparasitica 13: 179-244.

Eisa, A.A., Ammar, I.M.A., Radwan, H.S.H. \& El-Nabawi A. 1984. Larvicidal activity and development inhibition of the red flour beetle,Tribolium castaneum (Herbst) fed on insect growth regulators treated wheat flour. Minufiya J. Agric. 8: 405-414.

Faruki, S.I. 2005. Effects of UV-radiation on the growth and development of malathion-susceptible and multiresistant strains of Tribolium castaneum (Herbst) (Coleoptera : Tenebrionidae). Bangladesh J. Entomol. 25(2): 55-63.

Faruki, S.I. \& Khan, A.R. 1993. Potency of UV-radiation on Cadra cautella (Walker) (Lepidoptera : Phycitidae) larvae treated with Bacillus thuringiensis var. kurstaki Univ. J. Zool. Rajshahi Univ. 12: 73-79.

Faruki SI, Parween S and Begum M. 2002. Potentiality of Baycidal on the growth and development of malathionsucceptible and multi-resistant strains of Tribolium castaneum (Herbst) treated at different larval ages. Univ. J. Zool. Rajshahi Univ. 21: 37-39 pp.

Faruki, S.I, Das, D.R. \& Khatun, S. 2005. Effects of UVradiation on the larvae of the lesser mealworm, Alphitobius diaperinus (Panzer) (Coleoptera : Tenebrionidae) and their progeny. J. bio. sci. 5(4): 444-448.

Faruki, S.I, Das, D.R., Khan, A. R \&. Khatun, M. 2007. Effects of ultraviolet $(254 \mathrm{~nm})$ irradiation on egg hatching and adult emergence of the flour beetles, Tribolium castaneum, T. confusum and the almond moth, Cadra cautella. J. Insect Sci. 7: 36.

Fox, P. 1990. Insect Growth Regulators. PJB Publ. Ltd. Richmond. UK. 102 pp.

Hinton, H.E. \& Corbet, A.B. 1975. Common Insect pests of stored products. London Trustees of the British Museum (Natural History) 15: 39 pp.

Ichinose, T., Shibazaki, S. \& Ohata, M. 1980. Studies on the biology and mode of infestation of the tenebrionid beetle, Alphitobius diaperinus (Panzer) Harmful to broiler chicken houses. Japan J. Appl. Entomol. Zool. 20: $167-174$. 
McAllister, J.C., Steelman, C.D. \& Skeeles, J.K. 1994. Reservoir competence of the lesser mealworm (Coleoptera : Tenebrionidue) for Salmonella typhimurium (Eubacteriales : Enterobacteriaceae). $J$. Med. Entomol. 31: 369-372.

Mian, L.S. \& Mulla, M.S. 1982. Biological activity of IGRs against four stored product coleopteras. J. Econ. Entomol. 75: 80-85.

Mondal, K.A.M.S.H. \& Port, G.R. 1995. Effect of cyromazine o larval growth and adult population of susceptible and malathion-resistant strains of Tribolium castaneum Herbst. J. bio. sci. 3: 1-10.

Park, T. \& Frank, M.B.1948. The fecundity and development of flour beetles Tribolium confusum and Tribolium castaneum of three constant temperature. Ecology 29: 368-375.

Parween, S. 2003. Embryocidal and larvicidal effects of triflumuron against the red flour beetle, Tribolium castaneum (Herbst.) (Coleoptera: Tenebrionidae). Int. Pest Control 45 (6): 329-332.

Parween, S. 2004. Triflumuron: a potent candidate for the control of two strains of Tribolium castaneum Herbst (Coleopteran: Tenebrionidae). J. bio-sci. 12: 7-14.

Parween, S., Faruki, S.I \& Begum, M. 2001. Impairment of reproduction in the red flour beetle, Tribolium castaneum (Herbst) (Coleoptera : Tenebrionidae) due to larval feeding on triflumuron treated diet. J. Appl. Entomol. 125: 413-416.

Parween, S., Faruki, S.I. \& Akther, R. 2004. Growth and development of Alphitobius diaperinus (Panzer) (Coleoptera : Tembriomidae) Developing from pupae irradiated with ultra-violet rays. Univ. j. zool. Rajshahi Univ. 23: 23-26.

Pfeiffer, D.G. \& Axtell, R.C. 1980. Coleoptera of poultry manure in caged-layer in North Carolina. Environ. Entomol. 42: 575-577.

Salin, C., Delettre, Y.R. \& Vernon, P. 2003. Controlling the Mealworm Alphitobius diaperinus (Coleoptera : Tenebrionidae) in Broiler and Turkey Houses : Field Trials with a Combined Insecticides Treatment : Insect Growth Regulator and Pyrethroid. J. Econ. Entomol. 96 (1): $126-130$.

Schroeckenstein, D.C., Dans, S.M. \& Bush, R.K. 1990. Occupational Sensitivity to Tenebrio molitor Linnaeus (Yellow mealworm). J. Allergy and Clinical Immunol. 86 (2): 182-188.

Thomas, P.J. \& Bhatnagar-Thomas, P.L. 1968. Use of a juvenile hormone analogue as insecticide for pests of stored grain. Nature 219: 949p.

Manuscript received on 01.10.07, accepted on 08.11.07 